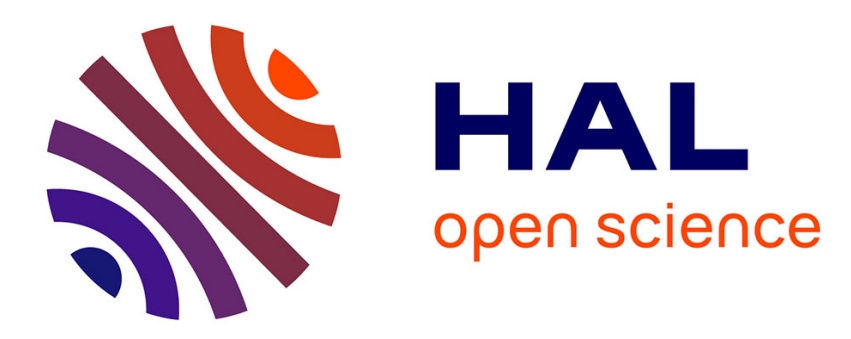

\title{
Optimal consumption and investment under time-varying relative risk aversion
}

Mogens Steffensen

\section{To cite this version:}

Mogens Steffensen. Optimal consumption and investment under time-varying relative risk aversion. Journal of Economic Dynamics and Control, 2011, 35 (5), pp.659. 10.1016/j.jedc.2010.12.007 . hal00796302

\section{HAL Id: hal-00796302 \\ https://hal.science/hal-00796302}

Submitted on 3 Mar 2013

HAL is a multi-disciplinary open access archive for the deposit and dissemination of scientific research documents, whether they are published or not. The documents may come from teaching and research institutions in France or abroad, or from public or private research centers.
L'archive ouverte pluridisciplinaire HAL, est destinée au dépôt et à la diffusion de documents scientifiques de niveau recherche, publiés ou non, émanant des établissements d'enseignement et de recherche français ou étrangers, des laboratoires publics ou privés. 


\section{Author's Accepted Manuscript}

Optimal consumption and investment under timevarying relative risk aversion

Mogens Steffensen

PII:

DOI:

S0165-1889(10)00278-2

Reference:

doi:10.1016/j.jedc.2010.12.007

DYNCON 2516

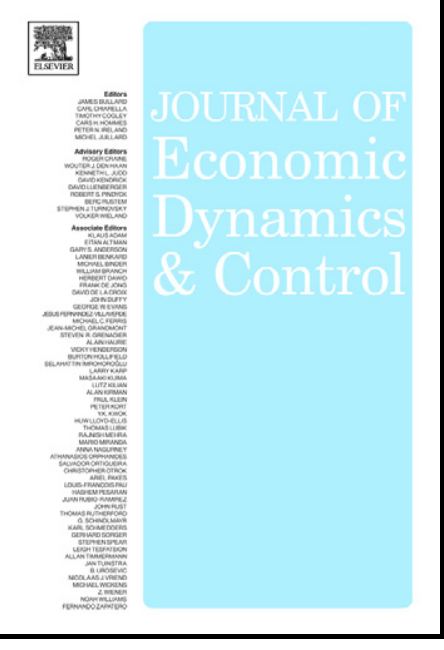

To appear in: Journal of Economic Dynamics

www.elsevier.com/locate/jedc \& Control

Received date: 19 November 2009

Accepted date: 3 December 2010

Cite this article as: Mogens Steffensen, Optimal consumption and investment under time-varying relative risk aversion, Journal of Economic Dynamics \& Control, doi:10.1016/j.jedc.2010.12.007

This is a PDF file of an unedited manuscript that has been accepted for publication. As a service to our customers we are providing this early version of the manuscript. The manuscript will undergo copyediting, typesetting, and review of the resulting galley proof before it is published in its final citable form. Please note that during the production process errors may be discovered which could affect the content, and all legal disclaimers that apply to the journal pertain. 


\title{
Optimal Consumption and Investment
}

\section{under Time-Varying Relative Risk Aversion}

\author{
Mogens Steffensen* \\ Department of Mathematical Sciences \\ University of Copenhagen
}

\begin{abstract}
We consider the continuous time consumption-investment problem originally formalized and solved by Merton in case of constant relative risk aversion. We present a complete solution for the case where relative risk aversion with respect to consumption varies with time, having in mind an investor with age-dependent risk aversion. This provides a new motivation for life-cycle investment rules. We study the optimal consumption and investment rules, in particular in the case where the relative risk aversion with respect to consumption is increasing with age.

JEL code: G11

Keywords: Merton's problem; Hamilton-Jacobi-Bellman equation; marginal indirect utility; life-cycle investment
\end{abstract}

*Address: Department of Mathematical Sciences, Universitetsparken 5, DK-2100 Copenhagen, Denmark; Phone: 4535320789; Mail: mogens@math.ku.dk 


\section{Introduction}

In classical consumption-investment problems with constant relative risk aversion with respect to consumption the optimal demand for stocks is constant, see Merton $(1969,1971,1990)$. There are several ways to generalize the problem such that the solution deviates from this simple rule. In this article we return to the original consumption-investment problem and account for a possibly time-varying risk aversion with respect to consumption. By this we mean that we allow the curvature of the instantaneous utility function with respect to consumption to vary with time. Henceforth, we speak of this as time-varying risk aversion although the Arrow-Pratt risk aversion was originally introduced in connection with the indirect utility of wealth rather than consumption.

The primary example is an individual with a decreasing risk aversion over time or, equivalently, with age. This appears to be a plausible motivation for time-dependence in portfolios. Many authors have studied the evidence for variation in risk tolerance in relation to age and other characteristics like gender, education etc. Recently, Al-Ajmi (2008) and Ho (2009) found evidence that risk aversion increases with age. Although we have increasing risk aversion in mind, we emphasize that our results hold for general time-variation. A related study based on different techniques can be found in Aase (2009).

Time-varying risk aversion with respect to consumption or wealth has certainly been studied on macro-level in connection with formation of asset prices, see e.g. Li (2007), and in connection with fluctuations in wealth and consumption, see e.g. Brunnermeier and Nagel (2008). Habit formation and generalized investment opportunities may also result in timevarying or possibly even stochastic risk aversion with respect to wealth, see e.g. Campbell and Viceira (2002) and Munk (2008). However, we go back to Merton's original formulation and model time-variation directly via the investor's preferences over the life-cycle: How to invest when the shape of your instantaneous utility function follows the shape of your back? 
We choose to present the result in the simple Black-Scholes-Merton market in order to stay focused on the main idea. Generalizations to include multidimensional complete markets and/or mortality is straightforward once this idea is understood. The idea is simple and the source of inspiration is Lakner and Nygren (2006). They solve a different intertemporal choice problem where they use that the marginal indirect utility obtained from intermediate consumption and terminal wealth is the same even in the case where the utility functions are different.

We approach the problem by dynamic programming and establish a Hamilton-JacobiBellman equation that characterizes the value function of the problem. Motivated by Lakner and Nygren (2006) we are able to come up with a solution to the Hamilton-Jacobi-Bellman equation that leads us to the optimal consumption rate and demand for stocks. The solution is, of course, more involved than in the classical case with constant relative risk aversion. Actually, the separation of the value function in two functions relating to the state variables time and wealth, respectively, does not hold.

Our main result is a complete characterization of the optimal consumption rate and demand for stocks for an investor with time-varying relative risk aversion. Furthermore, we study the behavior of the optimal decision rules and derive the dynamics of the optimal demand for stocks. The complete characterization is shown to specialize to Merton's classical result in case of constant relative risk aversion. In case of increasing risk aversion with age, we find that the optimal consumption rate is a convex function of wealth. Moreover, we show how the optimal demand for stocks increases with wealth. We learn that, for increasing risk aversion, the optimal demand for stocks from investors who are globally less risk-averse than the log-utility investor, has a negative drift. Also investors who are globally more risk averse than the log-utility investor have a globally decreasing optimal demand for stocks but their optimal demand has a locally positive drift in times where the optimal consumption 
rate is relatively low.

The paper is organized as follows. In Section 2 we present the decision problem and a characterization of the solution via a Hamilton-Jacobi-Bellman equation. In Section 3 we explain how the solution is constructed based on the ideas of Lakner and Nygren (2006). In Section 4 we verify that our candidate for the value function is actually the value function and present the optimal controls. In Section 5 we present Merton's solution as a special case of our results. In Section 6 we study the optimal decision rules in more detail and conclude.

\section{The decision problem and a characterization of its solution}

We assume that the individual operates in a standard Black-Scholes-Merton financial market with two assets, a bond $(B)$ and a stock $(S)$ the dynamics of which are given by

$$
\begin{aligned}
& d B(t)=r B(t) d t, \\
& B(0)=1, \\
& d S(t)=S(t)(\alpha d t+\sigma d W(t)), \\
& S(0)=s_{0},
\end{aligned}
$$

where $r, \alpha$ and $\sigma$ are constants and $W$ is a Brownian motion.

We denote the demand for stocks by $\pi$ and the consumption rate by $c$ such that the dynamics of the wealth of an investor with initial wealth $x_{0}$ are given by

$$
\begin{aligned}
d X(t) & =(r+\pi(t)(a-r)) X(t) d t+\pi(t) \sigma X(t) d W(t)-c(t) d t \\
X(0) & =x_{0} .
\end{aligned}
$$

We consider the objective to maximize expected utility of future consumption until the end 
of a deterministic time horizon $T$,

$$
\sup _{(c, \pi) \in \mathcal{A}} E\left[\int_{0}^{T} F(t, c(t)) d t\right],
$$

and for this we introduce the value function

$$
V(t, x)=\sup _{(c, \pi) \in \mathcal{A}} E_{t, x}\left[\int_{t}^{T} F(s, c(s)) d s\right] .
$$

Here $\mathcal{A}$ is a set of admissible controls which essentially means that they admit a unique non-negative solution to (1).

More specifically we focus on the case that can be formalized by

$$
F(t, c)=\frac{1}{1-\gamma(t)} w^{\gamma(t)}(t) c^{1-\gamma(t)}
$$

Here, $\gamma$ is a deterministic time-varying relative risk aversion. If $\gamma$ is constant we are in the classical case of constant relative risk aversion. The function $w$ is a deterministic positive time weight function that we for notational convenience take to the power of $\gamma$ without loss of generality. The special case with constant rate of time preference $\rho$ can be specified by letting $w$ be defined by

$$
w^{\gamma(t)}(t)=e^{-\rho t}
$$

We emphasize that the time-variation of $w$, essentially corresponding to time-variation of the subjective discount factor, is just introduced for the sake of generality. It is the timevariation of $\gamma$ which is central for what follows. We focus on the finite time horizon problem. However, the solution to the infinite time horizon problem can essentially be found by letting $T$ go to infinity below. Note, however, that in the infinite time horizon problem, we expect that the optimal controls remain time-varying, even for a constant rate of time preference, due to the time-variation of risk aversion.

The value function is characterized by the so-called Hamilton-Jacobi-Bellman equation. Denoting partial derivatives by subscript, the result states that if we have a sufficiently 
regular solution to the system,

$$
\begin{aligned}
V_{t}(t, x)+\sup _{c, \pi}\left[((r+\pi(\alpha-r)) x-c) V_{x}(t, x)+\frac{1}{2} \sigma^{2} \pi^{2} x^{2} V_{x x}(t, x)+F(t, c)\right] & =0,(5) \\
V(T, x) & =0,
\end{aligned}
$$

then this solution is indeed the value function and the optimal control functions are given by

$$
\arg \sup _{c, \pi}\left[((r+\pi(\alpha-r)) x-c) V_{x}(t, x)+\frac{1}{2} \sigma^{2} \pi^{2} x^{2} V_{x x}(t, x)+F(t, c)\right],
$$

with $F$ specified in (3).

Taking derivative under the square brackets in (5) with respect to $c$ and $\pi$, respectively, and equating to zero gives the following functions as candidates for the optimal control functions,

$$
\begin{aligned}
c^{*}(t, x) & =w(t) V_{x}^{-\phi(t)}(t, x), \\
\pi^{*}(t, x) & =-\frac{\alpha-r}{\sigma^{2}} \frac{V_{x}(t, x)}{x V_{x x}(t, x)},
\end{aligned}
$$

where we have introduced the notation

$$
\phi(t)=\gamma^{-1}(t)
$$

The optimizers $c^{*}$ and $\pi^{*}$ are indeed maximizers if the double derivatives under the square brackets are negative leading to the conditions,

$$
\begin{aligned}
-\gamma(t) w^{\gamma(t)}(t) c^{-\gamma(t)-1} & <0 \\
V_{x x}(t, x) & <0
\end{aligned}
$$

which has to be checked at the end.

Plugging the control functions back into the Hamilton-Jacobi-Bellman equation gives the 
following partial differential equation,

$$
\begin{aligned}
V_{t}(t, x)+r x V_{x}(t, x)-\frac{1}{2} \theta^{2} \frac{V_{x}^{2}(t, x)}{V_{x x}(t, x)}-w(t) \frac{1}{1-\phi(t)} V_{x}^{1-\phi(t)}(t, x) & =0 \\
V(T, x) & =0
\end{aligned}
$$

where we have abbreviated the market price of risk,

$$
\theta=\frac{\alpha-r}{\sigma}
$$

To proceed from here we need a qualified guess on the solution to (8). In the classical case of constant relative risk aversion, we assume separability of $V$ and end up with a value function in the form

$$
V(t, x)=\frac{1}{1-\gamma} f^{\gamma}(t) x^{1-\gamma},
$$

for a deterministic function $f$. With time-varying risk aversion, separability does not hold and in the next section we provide the economical motivation for the generalized solution that replaces $(9)$.

\section{Motivation for a qualified guess}

In this section we motivate a better guess on (8) than (9). We stress that the arguments in this section are informal. We do not have to account for all details here since this is just what puts us on the right track. The formal verification that our guess indeed solves (8) is provided in Section 4.

To come up with a better guess we get our inspiration from Lakner and Nygren (2006). They solve a problem which combines utility of consumption with utility of terminal wealth. The important part of their argument is that they allocate the initial wealth into one part for intermediate consumption and another part for terminal wealth. They then solve each of the two problems separately. The fact that each of the two problems are actually formulated 
as constrained problems plays no role for us. Finally, they determine the optimal allocation of initial wealth such that the marginal indirect utility from the two problems coincide. This algorithm is found to hold, even when the functions that measure utility from consumption and terminal wealth, differ. This inspires to a qualified guess on a solution to our problem with time-dependent risk aversion.

We guess that the value function can be constructed in the following way. At time 0 we allocate the initial wealth into a continuum of infinitesimal terminal wealth problems without intermediate consumption. For all time points $0<s \leq T$, we allocate an infinitesimal initial capital $x_{0}^{s}$ which we determine later on, to consumption at time $s$. For every problem in this continuum of problems, we let the wealth develop similarly to the wealth dynamics in (1) with an investment strategy $\pi^{s}$ and no consumption. We then consider the investor's problem to maximize utility of time $s$ terminal wealth,

$$
\sup _{\pi^{s}} E\left[w^{\gamma(s)}(s) \frac{1}{1-\gamma(s)} X^{s}(s)^{1-\gamma(s)}\right]
$$

The solution to the time $s$ optimization problem (10) is related to the following value function $V^{s}$

$$
V^{s}\left(t, x^{s}\right)=\frac{1}{1-\gamma(s)} f^{s}(t)^{\gamma(s)}\left(x^{s}\right)^{1-\gamma(s)}, t \leq s
$$

see Merton (1990). Note that this problem is namely blind to the time-variation of relative risk aversion between time 0 and time $s$. Only the relative risk aversion at the terminal time point $s$ matters when there is no intermediate consumption between time 0 and time $s$.

The question is now how to allocate the initial wealth $x$ to this continuum of terminal wealth problems and here we assume that the idea of Lakner and Nygren (2006) holds in our case. We equate the marginal indirect utility from this continuum of infinitesimal investment problems such that $V_{x}^{s}\left(t, x^{s}\right)$ is constant in $s$ but not in $t$ and the total wealth $x$. I.e., we 
assume that there exists a function $k(t, x)$ such that

$$
V_{x}^{s}\left(t, x^{s}\right)=f^{s}(t)^{\gamma(s)}\left(x^{s}\right)^{-\gamma(s)}=k(t, x)
$$

By isolating $x^{s}$ in (12) we can then represent the allocation $x^{s}$ in terms of the marginal indirect utility $k$ which is yet to be determined,

$$
x^{s}=f^{s}(t) k(t, x)^{-\phi(s)}
$$

Our hypothesis is that (13) forms the optimal capital allocation to the time $s$ consumption. Plugging this allocation into (11) gives us the value function $V$ in terms of $k$,

$$
\begin{aligned}
V(t, x) & =\int_{t}^{T} V^{s}\left(t, x^{s}\right) d s \\
& =\int_{t}^{T} \frac{1}{1-\gamma(s)} f^{s}(t)^{\gamma(s)}\left(x^{s}\right)^{1-\gamma(s)} d s \\
& =\int_{t}^{T} \frac{1}{1-\gamma(s)} f^{s}(t) k(t, x)^{1-\phi(s)} d s .
\end{aligned}
$$

What remains is to determine $k$. But this is determined by the budget constraint that the continuum of allocations has to sum up to $x$,

$$
x=\int_{t}^{T} x^{s} d s=\int_{t}^{T} f^{s}(t) k(t, x)^{-\phi(s)} d s .
$$

In general, we are not able to isolate $k$ in the non-linear equation (15). But for a concrete function $\phi$, the function $k$ can easily be determined by approximating the integral. This concludes our motivation for guessing that the solution takes the explicit form (14) with $k$ determined by the non-linear equation (15). We emphasize that we need not to show that the arguments above inspired by Lakner and Nygren (2006) formally hold in our case. They just lead us to our guess which now has to be formally verified.

\section{Verification and optimal controls}

We recapitulate that our guess on $V$ is formed by the system of equations, 


$$
\begin{aligned}
V(t, x) & =\int_{t}^{T} \frac{1}{1-\gamma(s)} f^{s}(t) k(t, x)^{1-\phi(s)} d s, \\
x & =\int_{t}^{T} f^{s}(t) k(t, x)^{-\phi(s)} d s .
\end{aligned}
$$

We assume that there exists a unique solution to this system. Furthermore, we assume sufficient differentiability for all operations below to be valid. Both existence of a unique solution and differentiability below relies on a sufficiently regular relative risk aversion function $\gamma$ and on a sufficiently regular weight function $w$. A sufficient condition which is typically fulfilled, is that they are continuous and bounded from above and from below away from zero.

First we concentrate on the derivatives in $t$. Introducing a function $h$ defined as

$$
h(t, x)=\int_{t}^{T} \phi(s) f^{s}(t) k(t, x)^{-\phi(s)} d s,
$$

we form the partial derivative $V_{t}$ in terms of $k$ and $k_{t}$ from (16),

$V_{t}(t, x)=-\frac{1}{1-\gamma(t)} f^{t}(t) k(t, x)^{1-\phi(t)}+\int_{t}^{T} \frac{1}{1-\gamma(s)} f_{t}^{s}(t) k(t, x)^{1-\phi(s)} d s-k_{t}(t, x) h(t, x)$.

We then form the partial derivative $k_{t}$ by differentiating with respect to $t$ on both sides of $(17)$

$$
\begin{aligned}
0 & =-f^{t}(t) k(t, x)^{-\phi(t)}+\int_{t}^{T} f_{t}^{s}(t) k(t, x)^{-\phi(s)} d s-\frac{k_{t}(t, x)}{k(t, x)} h(t, x) \Rightarrow \\
k_{t}(t, x) & =\frac{-f^{t}(t) k(t, x)^{1-\phi(t)}+\int_{t}^{T} f_{t}^{s}(t) k(t, x)^{1-\phi(s)} d s}{h(t, x)} .
\end{aligned}
$$

Plugging (20) into (19) gives

$$
V_{t}(t, x)=\frac{1}{1-\phi(t)} f^{t}(t) k(t, x)^{1-\phi(t)}-\int_{t}^{T} \frac{1}{1-\phi(s)} f_{t}^{s}(t) k(t, x)^{1-\phi(s)} d s .
$$

Now we turn to the derivatives in $x$. We form the partial derivative $V_{x}$ in terms of $k$ and $k_{x}$ from (16),

$$
V_{x}(t, x)=-k_{x}(t, x) h(t, x)
$$


We then form the partial derivative $k_{x}$ by differentiating with respect to $x$ on both sides of $(17)$

$$
\begin{aligned}
1 & =k_{x}(t, x) \int_{t}^{T} \phi(s) f^{s}(t) k(t, x)^{-\phi(s)-1} d s=-\frac{k_{x}(t, x)}{k(t, x)} h(t, x) \Rightarrow \\
k_{x}(t, x) & =-\frac{k(t, x)}{h(t, x)}
\end{aligned}
$$

Plugging (23) into (22) gives

$$
V_{x}(t, x)=k(t, x)
$$

and, consequently,

$$
V_{x x}(t, x)=k_{x}(t, x)
$$

Note here the connection between the role of $k$ in our motivation for the guess in Section 3 , and the relation (24). The function $k$ was introduced as the marginal indirect utility for each of the continuum of terminal wealth problems leading to $V$. From (24) we learn that this coincides with the total marginal indirect utility.

We are now, finally, ready to plug (21), (24), and (25) into (8) to get

$$
\begin{aligned}
0= & \overbrace{\frac{1}{1-\phi(t)} f^{t}(t) k(t, x)^{1-\phi(t)}-w(t) \frac{1}{1-\phi(t)} k(t, x)^{1-\phi(t)}}^{\left(1^{\prime}\right)} \\
& \underbrace{-\int_{t}^{T} \frac{1}{1-\phi(s)} f_{t}^{s}(t) k(t, x)^{1-\phi(s)} d s+r x k(t, x)-\frac{1}{2} \theta^{2} \frac{k^{2}(t, x)}{k_{x}(t, x)}}_{\left(2^{\prime}\right)} .
\end{aligned}
$$

First, we note that in $(26),\left(1^{\prime}\right)=0$ if

$$
f^{t}(t)=w(t)
$$

Second, we note that, by $(17), x k(t, x)$ in $\left(2^{\prime}\right)$ can be written as

$$
\int_{t}^{T} f^{s}(t) k(t, x)^{1-\phi(s)} d s
$$

Third, we note that, by (23), $\frac{k^{2}(t, x)}{k_{x}(t, x)}$ in $\left(2^{\prime}\right)$ can be written as

$$
-h(t, x) k(t, x)=-\int_{t}^{T} \phi(s) f^{s}(t) k(t, x)^{1-\phi(s)} d s .
$$


We conclude then that $\left(2^{\prime}\right)=0$ can be written as

$$
\int_{t}^{T}\left(r f^{s}(t)+\frac{1}{2} \theta^{2} \phi(s) f^{s}(t)-\frac{1}{1-\phi(s)} f_{t}^{s}(t)\right) k(t, x)^{1-\phi(s)} d s=0 .
$$

However, this is seen to be true if

$$
f_{t}^{s}(t)=(1-\phi(s))\left(r+\frac{1}{2} \theta^{2} \phi(s)\right) f^{s}(t)
$$

This ordinary differential equation in $f$ together with (27) has the solution

$$
f^{s}(t)=e^{-(1-\phi(s))\left(r+\frac{1}{2} \theta^{2} \phi(s)\right)(s-t)} w(s) .
$$

Note that $\phi(s)$ rather than $\phi(t)$ appears in the differential equation for $f$. This relates to the fact that all of the terminal wealth problem that forms the continuum on which our guess is based, are blind to time-variation of relative risk aversion and sees only the relative risk aversion at the horizon $s$.

This concludes the verification that our guess (16) with (17) is indeed a solution to (8). What remains is to specify the optimal control functions and check the conditions (6) and (7). The optimal control functions are

$$
\begin{aligned}
c^{*}(t, x) & =w(t) V_{x}^{-\phi(t)}(t, x)=w(t) k(t, x)^{-\phi(t)} \\
\pi^{*}(t, x) & =-\frac{\alpha-r}{\sigma^{2}} \frac{V_{x}(t, x)}{x V_{x x}(t, x)}=-\frac{\alpha-r}{\sigma^{2}} \frac{k(t, x)}{x k_{x}(t, x)}=\frac{\alpha-r}{\sigma^{2}} \frac{h(t, x)}{x}
\end{aligned}
$$

The conditions (6) and (7) can now be written as

$$
\begin{aligned}
-\gamma(t) w^{\gamma(t)}(t) c^{-\gamma(t)-1} & =-\gamma(t) w(t)^{-1} k(t, x)^{\frac{1+\gamma(t)}{\gamma(t)}}<0, \\
V_{x x}(t, x) & =k_{x}(t, x)=-\frac{k(t, x)}{h(t, x)}<0 .
\end{aligned}
$$

Since $f$ if positive it follows directly from the definitions of these quantities that $k>0$ and $h>0$ such that the conditions are fulfilled.

Before we take a closer look at the optimal decision rules (29) and (30), it is time to check that the classical results by Merton appear as a special case. 


\section{Merton's solution as special case}

If we take $\gamma$ to be a constant, Merton's problem appears and we should get his classical result. But in this case the defining relations for $k$ and $h$ are

$$
\begin{aligned}
x & =k(t, x)^{-\phi} \int_{t}^{T} f^{s}(t) d s, \\
h(t, x) & =\phi k(t, x)^{-\phi} \int_{t}^{T} f^{s}(t) d s,
\end{aligned}
$$

such that

$$
\begin{aligned}
k(t, x) & =\left(\frac{x}{\int_{t}^{T} f^{s}(t) d s}\right)^{-\gamma} \\
h(t, x) & =\phi x .
\end{aligned}
$$

The value function and the optimal decision rules then turn into

$$
\begin{aligned}
V(t, x) & =\frac{1}{1-\gamma}\left(\int_{t}^{T} f^{s}(t) d s\right)^{\gamma} x^{1-\gamma}, \\
c(t, x) & =\frac{w(t)}{\int_{t}^{T} f^{s}(t) d s} x \\
\pi(t, x) & =\phi \frac{\alpha-r}{\sigma^{2}}
\end{aligned}
$$

that can be recognized as Merton's solution.

\section{The optimal decision rules}

We now take a closer look at the optimal decision rules. For a general function $\gamma$ one cannot say much about the behavior of the optimal decisions. For this section the hypothesis is that monotonicity of $\gamma$ is somehow inherited in the optimal decision rules. We focus on the case of increasing risk aversion but opposite effects could be obtained by assuming decreasing risk aversion. Increasing risk aversion was often reported but recent studies find that the effect of age on risk tolerance is more complex, see Al-Ajmi (2008) and references therein. 
When studying the behavior of the optimal decisions, it turns out convenient to introduce the following functions

$$
h_{n}(t, x)=\int_{t}^{T} \phi^{n}(s) f^{s}(t) k(t, x)^{-\phi(s)} d s,
$$

such that $h_{0}(t, x)=x, h_{1}(t, x)=h(t, x)$, and the following rule applies,

$$
\begin{aligned}
\frac{\partial}{\partial x} h_{0}(t, x) & =1 \\
\frac{\partial}{\partial x} h_{n}(t, x) & =\frac{h_{n+1}(t, x)}{h_{1}(t, x)}, n>1 .
\end{aligned}
$$

Hereafter, subscript after $h$ refers to (31) rather than partial derivative.

We start out by looking at the optimal consumption rate (29). Using (23) we can calculate the marginal optimal consumption rate,

$$
c_{x}^{*}(t, x)=\frac{\phi(t) w(t) k(t, x)^{-\phi(t)}}{h(t, x)} .
$$

Thus, this marginal consumption rate is positive as we would expect. It is more interesting to calculate the second order derivative in order to learn about the shape of consumption as a function of wealth and compare it with the linear rule in case of constant relative risk aversion. We find that

$$
c_{x x}^{*}(t, x)=\frac{\phi(t) w(t) k(t, x)^{-\phi(t)}}{h^{2}(t, x)}\left(\phi(t)-\frac{h_{2}(t, x)}{h_{1}(t, x)}\right) .
$$

From the definition of $h$ we realize that $\phi(t)-h_{2}(t, x) / h_{1}(t, x)$ is the difference between $\phi$ evaluated at time $t$ and a weighted average of future $\phi$ with weights given by $\phi(s) f^{s}(t) k(t, x)^{-\phi(s)}$. Thus, we can immediately conclude that this term is positive for increasing $\gamma$ corresponding to decreasing $\phi$. This means that for $\gamma$ increasing, the optimal consumption rate is a convex function of wealth.

We now focus on the optimal investment strategy. Plugging in the expression (17) for $x$ and the definition of $h$ we see that $\pi^{*}$ can be written in the following way,

$$
\pi^{*}(t, x)=\frac{\alpha-r}{\sigma^{2}} \frac{\int_{t}^{T} \phi(s) f^{s}(t) k(t, x)^{-\phi(s)} d s}{\int_{t}^{T} f^{s}(t) k(t, x)^{-\phi(s)} d s} .
$$


The last term forms a weighted average of future inverse relative risk aversion with weights given by $f^{s}(t) k(t, x)^{-\phi(s)}$. From this observation we can again immediately recognize Merton's proportion for constant relative risk aversion.

The optimal demand for stocks in terms of $h^{n}$ becomes

$$
\pi^{*}(t, x)=\frac{\theta}{\sigma} \frac{h_{1}(t, x)}{h_{0}(t, x)} .
$$

From this presentation and the rule (32) we can immediately calculate the marginal optimal demand for stocks,

$$
\pi_{x}^{*}(t, x)=\frac{\theta}{\sigma} \frac{\frac{h_{2}(t, x)}{h_{1}(t, x)}-\frac{h_{1}(t, x)}{h_{0}(t, x)}}{x} .
$$

From the definition of $h^{n}$ we realize that both ratios in the numerator are actually weighted averages of the same function $\phi$. However, in the first ratio, $h_{2} / h_{1}$, the weights from the second ratio, $h_{1} / h_{0}$, are multiplied by $\phi$. One has to be careful here, since $\phi$ is now both the average function and what actually differs the weights in the two averages. So, since the difference in weight function $\phi$ is a decreasing function for increasing $\gamma$, the average in the first ratio is formed with relatively more weight on the 'small' $s$ values than in the second ratio and, correspondingly, relatively less weight on 'large' $s$ values. Furthermore, since the average function $\phi$ is decreasing, the difference in weights means that the first ratio is larger than the second ratio, and we conclude that the numerator is positive. From this we conclude that the optimal demand for stocks increases in wealth for a fixed time point. This is an important observation in itself. We will also use the form (33) to derive the dynamics of $\pi$. In the Appendix we prove that they are, suppressing the argument $(t, X(t))$ of the functions $h_{n}$,

$$
\begin{aligned}
d \pi^{*}(t, X(t))= & \frac{\theta}{\sigma} \frac{c^{*}(t, X(t))}{X(t)}\left(\frac{h_{1}}{h_{0}}-\phi(t)\right) d t \\
& +\theta^{2}\left(\frac{h_{2}}{h_{1}}-\frac{h_{1}}{h_{0}}\right)\left(1-\frac{h_{1}}{h_{0}}\right) \pi^{*}(t, X(t)) d t \\
& +\theta\left(\frac{h_{2}}{h_{1}}-\frac{h_{1}}{h_{0}}\right) \pi^{*}(t, X(t)) d W(t)
\end{aligned}
$$


The three terms in the dynamics of $\pi^{*}$ in (34) have different interpretations. In the first term appears the expression $h_{1} / h_{0}-\phi(t)$. From the definition of $h$ we realize that this is the difference between a weighted average of future $\phi$ and $\phi$ evaluated at time $t$. Thus, we can immediately conclude that that this term is negative for increasing $\gamma$ corresponding to decreasing $\phi$. This term forms a direct first order effect from consumption. Inspired from the motivation for our guess we can say that the consumption at time $t$ is connected with investment at inverse relative risk aversion $\phi(t)$ whereas the residual wealth is reinvested in accordance with the future $\phi$. This gives a drift corresponding to the first term of (34).

In the second term in the dynamics for the optimal demand for stocks, (34), reappears the term $h_{2} / h_{1}-h_{1} / h_{0}$ from the marginal optimal demand $\pi_{x}^{*}$. Thus, we already argued that this term is positive. This term is now multiplied by $1-h_{1} / h_{0}$, where again $h_{1} / h_{0}$ is a weighted average of $\phi$. If $\gamma>1,1-h_{1} / h_{0}>0$ and the second part of the drift of $\pi^{*}$ is positive. Otherwise, if $\gamma<1,1-h_{1} / h_{0}<0$, and the second part of the drift of $\pi^{*}$ is negative. This term forms a second order effect from expected capital gains on wealth but can be positive or negative depending on the level of risk aversion.

In the third term in the dynamics for the optimal demand for stocks, (34), reappears again the term $h_{2} / h_{1}-h_{1} / h_{0}$. This is again positive and just reflects the diffusive effect on the optimal demand for stocks following from the diffusive capital gains.

Finally, we return to the observation above, that the second order effect can be positive or negative, depending on whether $\gamma>1$ or $\gamma<1$. We see that for $\gamma<1$ we always have a decreasing (expected) optimal demand for stocks since both terms of the drift of (34) are negative. For $\gamma>1$, however, the second term of the drift is positive and we do not have a generally decreasing (expected) optimal demand for stocks. Over the whole term of the decision problem the optimal demand does indeed decrease. This is clear since, for a 
sufficiently regular $\gamma$,

$$
\pi^{*}\left(0, x_{0}\right)=\frac{\theta}{\sigma} \frac{h_{1}\left(0, x_{0}\right)}{h_{0}\left(0, x_{0}\right)}>\frac{\theta}{\sigma} \phi(T)=\lim _{t \rightarrow T} \frac{\theta}{\sigma} \frac{h_{1}(t, X(t))}{h_{0}(t, X(t))}=\lim _{t \rightarrow T} \pi^{*}(t, X(t)) .
$$

Here the inequality follows since $h_{1} / h_{0}$ is a weighted average of future decreasing $\phi$ which is larger than the terminal value of the decreasing function $\phi$. But locally we can have an increasing (expected) optimal demand for stocks. This occurs in times where the second term in the drift of $\pi^{*}$ dominates the first term. This situation is easily obtained by a sufficiently low optimal consumption rate, here parametrized by a low weight function $w$. But as long as the first order effect from consumption dominates the second order effect from capital gains, the optimal demand for stock is decreasing for all investors.

The observations in this section contribute to the understanding of the connection between optimal demand for stocks and fluctuations in consumption and wealth. Our insight explains how fluctuations in risk aversion leads to fluctuations in the optimal demand for stocks partly as a consequence of fluctuations in wealth which is partly driven by fluctuations in consumption. But note that we do not take into account a possible reverse effect saying that fluctuations in wealth should have an effect on risk aversion. This would require explicit modelling of dependence of $\gamma$ on e.g. wealth. However, results by Brunnermeier and Nagel (2008) suggest that such effects do not exist.

Going back to the second order effect, it may appear counter-intuitive that we can construct a situation where investors with relatively high risk aversion increase their optimal demand for stocks while investors with relatively low risk aversion decrease their optimal demand for stocks. However, this is just an optical illusion since the initial demand for stocks by the 'more risky' investor is larger than the initial optimal demand for stocks by the 'less risky' investor. And even after the reverse corrections of holdings at time $t$, the 'more risky' investor will, of course, have a higher optimal demand for stocks than will the 'less risky' investor. 
Moreover, it may appear counter-intuitive that the second order effect does not have the same qualitatively influence on all investors. However, this may have to do with the well-known fact that, in our problem formulation, we do actually not distinguish between relative risk aversion and elasticity of intertemporal substitution. Actually, an increasing $\gamma$ gives immediately a decreasing elasticity of infinitesimal intertemporal substitution $\phi$.

We have here presented a complete solution to Merton's problem with time-varying relative risk aversion. Allowing the risk aversion to increase with age provides a new motivation for life-cycle investment. Inspired by Lakner and Nygren (2006) we have learned what replaces the usual separation of variables in the value function. This is important insight in a classical problem. Furthermore, the explicit solution gives access to studying the optimal decision rules explicitly. However, there are still important things to be discussed, in particular in relation to recursive utility and incomplete markets. We propose that future research clarifies if and how the approach presented in this article can be generalized to recursive utility. By this we mean a problem with time-varying relative risk aversion and time-varying elasticity of infinitesimal intertemporal substitution. Furthermore, we propose that future research clarifies if and how our approach can be generalized to an incomplete market setting. For both recursive utility and incomplete markets, the idea from Lakner and Nygren (2006) does not apply directly from an intuitive point of view. For recursive utility the continuum of infinitesimal terminal wealth problems that leads us to our guess, are linked via the recursion and cannot be solved separately. For the incomplete market additional information from unhedgeable risk requires reallocation of capital between the different future time horizons over time. Adaptation of the solution presented in this article to these situations are topics for future research. 


\section{References}

Aase, K. K. (2009). The Investment Horizon Problem: A Resolution. Technical report, Available at SSRN: http://ssrn.com/abstract $=1496846$.

Al-Ajmi, J. (2008). Risk tolerance of individual investors in an emerging market. International Research Journal of Finance and Economics, 17, 15-26.

Brunnermeier, M. K. \& Nagel, S. (2008). Do Wealth Fluctuations Generate Time-Varying Risk Aversion? Micro-evidence on Individuals. American Economic Review, 98(3), $713-736$.

Campbell, J. \& Viceira, L. (2002). Strategic Asset Allocation. Oxford University Press.

Ho, H. (2009). An experimental study of risk aversion in decision-making under uncertainty. International Advances in Economic Research, 15(4), 369-377.

Lakner, P. \& Nygren, L. M. (2006). Portfolio Optimization with Downside Constraints. Mathematical Finance, 16(2), 283-299.

Li, G. (2007). Time-varying risk aversion and asset prices. Journal of Banking and Finance, $31,243-257$.

Merton, R. C. (1969). Lifetime Portfolio Selection under Uncertainty: The Continuous Time Case. Review of Economics and Statistics, 51, 247-257.

Merton, R. C. (1971). Optimum Consumption and Portfolio Rules in a Continuous Time Model. Journal of Economic Theory, 3, 373-413; Erratum 6 (1973); 213-214.

Merton, R. C. (1990). Continuous-time Finance. Blackwell. 
Munk, C. (2008). Portfolio and consumption choice with stochastic investment opportunities and habit formation in preferences. Journal of Economic Dynamics and Control, 32(11), $3560-3589$.

\section{Appendix}

Proof of (34): From (33) and (32) we calculate the second order partial derivative of $\pi^{*}$ in $x$, here suppressing the argument $(t, x)$ everywhere,

$$
\begin{aligned}
\pi_{x x}^{*} & =\frac{\theta}{\sigma} \frac{x\left(\frac{h_{3}}{h_{1}^{2}}-\frac{h_{2}^{2}}{h_{1}^{3}}-\frac{h_{2}}{h_{0} h_{1}}+\frac{h_{1}}{h_{0}^{2}}\right)-\left(\frac{h_{2}}{h_{1}}-\frac{h_{1}}{h_{0}}\right)}{x^{2}} \\
& =\frac{\theta}{\sigma} \frac{\frac{h_{0} h_{3}}{h_{1}^{2}}-\frac{h_{0} h_{2}^{2}}{h_{1}^{3}}-2 \frac{h_{2}}{h_{1}}+2 \frac{h_{1}}{h_{0}}}{x^{2}} .
\end{aligned}
$$

More calculations lead, via (20), (29), and (28), to the partial derivative of $\pi^{*}$ in $t$,

$$
\begin{aligned}
\pi_{t}^{*}= & \frac{\theta}{\sigma} \frac{\frac{\partial}{\partial t} h}{x} \\
= & -\phi(t) f^{t}(t) k(t, x)^{-\phi(t)} \\
& +\int_{t}^{T} \phi(s) f_{t}^{s}(t) k(t, x)^{-\phi(s)} d s-\frac{k_{t}(t, x)}{k(t, x)} \int_{t}^{T} \phi^{2}(s) f^{s}(t) k(t, x)^{-\phi(s)} d s \\
= & \frac{\theta}{\sigma} \frac{c^{*}\left(\frac{h_{2}}{h_{1}}-\phi(t)\right)+\int_{t}^{T}\left(\phi(s)-\frac{h_{2}}{h_{1}}\right) f_{t}^{s}(t) k^{-\phi(s)} d s}{x} \\
= & \frac{\theta}{\sigma} \frac{c^{*}\left(\frac{h_{2}}{h_{1}}-\phi(t)\right)+r h_{0}\left(\frac{h_{1}}{h_{0}}-\frac{h_{2}}{h_{1}}\right)+\frac{1}{2} \theta^{2}\left(\frac{h_{2}^{2}}{h_{1}}-h_{3}\right)}{x} .
\end{aligned}
$$


From the derivatives of $\pi^{*}$ we can go to the dynamics of $\pi^{*}$ via a plain Ito calculation,

$$
\begin{aligned}
& d \pi^{*}(t, X(t)) \\
= & \pi_{t}^{*}(t, X(t)) d t+\pi_{x}^{*}(t, X(t)) d X(t)+\frac{1}{2} \pi_{x x}^{*}(t, X(t)) \sigma^{2} \pi^{* 2}(t, X(t)) X^{2}(t) \\
= & \frac{\theta}{\sigma} \frac{c^{*}(t, X(t))\left(\frac{h_{2}}{h_{1}}-\phi(t)\right)+r h_{0}\left(\frac{h_{1}}{h_{0}}-\frac{h_{2}}{h_{1}}\right)+\frac{1}{2} \theta^{2}\left(\frac{h_{2}^{2}}{h_{1}}-h_{3}\right)}{X(t)} d t \\
& +\frac{\theta}{\sigma} \frac{\frac{h_{2}}{h_{1}}-\frac{h_{1}}{h_{0}}}{X(t)}\left(\left(r+\pi^{*}(t, X(t))(a-r)\right) X(t)-c^{*}(t, X(t))\right) d t \\
& +\frac{\theta}{\sigma} \frac{\frac{h_{2}}{h_{1}}-\frac{h_{1}}{h_{0}}}{X(t)} \pi^{*}(t, X(t)) \sigma X(t) d W(t) \\
& +\frac{1}{2} \frac{\theta}{\sigma} \frac{\frac{h_{0} h_{3}}{h_{1}^{2}}-\frac{h_{0} h_{2}^{2}}{h_{1}^{3}}-2 \frac{h_{2}}{h_{1}}+2 \frac{h_{1}}{h_{0}}}{X^{2}(t)} \sigma^{2} \pi^{* 2}(t, X(t)) X^{2}(t) d t .
\end{aligned}
$$

Now, after inserting $\pi^{*}(t, X(t))$ in the drift, a lot of terms vanish and we are left with

$$
\begin{aligned}
d \pi^{*}(t, X(t))= & \frac{\theta}{\sigma} \frac{c^{*}(t, X(t))}{X(t)}\left(\frac{h_{1}}{h_{0}}-\phi(t)\right) d t \\
& +\frac{\theta^{3}}{\sigma}\left(\frac{h_{2}}{h_{1}}-\frac{h_{1}}{h_{0}}\right) \frac{h_{1}}{h_{0}}\left(1-\frac{h_{1}}{h_{0}}\right) d t \\
& +\theta\left(\frac{h_{2}}{h_{1}}-\frac{h_{1}}{h_{0}}\right) \pi^{*}(t, X(t)) d W(t) .
\end{aligned}
$$

Inserting $\pi^{*}$ back into the second line of (35), we reach at (34). 